\title{
Machine Learning Paradigms for Modeling Spatial and Temporal Information in Multimedia Data Mining
}

\author{
Djamel Bouchaffra, ${ }^{1}$ Abbes Amira, ${ }^{2}$ Ce Zhu, ${ }^{3}$ and Chu-Song Chen ${ }^{4}$ \\ ${ }^{1}$ Grambling State University, Grambling, LA 71245, USA \\ ${ }^{2}$ Brunel University, Uxbridge, Middlesex UB83PH, UK \\ ${ }^{3}$ Nanyang Technological University, Singapore 639798 \\ ${ }^{4}$ Institute of Information Science, Taipei 115, Taiwan \\ Correspondence should be addressed to Djamel Bouchaffra, dbouchaffra@ieee.org
}

Received 31 December 2010; Accepted 31 December 2010

Copyright (C) 2010 Djamel Bouchaffra et al. This is an open access article distributed under the Creative Commons Attribution License, which permits unrestricted use, distribution, and reproduction in any medium, provided the original work is properly cited.

Multimedia data mining and knowledge discovery is a fast emerging interdisciplinary applied research area. There is tremendous potential for effective use of multimedia data mining (MDM) through intelligent analysis. Diverse application areas are increasingly relying on multimedia understanding systems. Advances in multimedia understanding are related directly to advances in signal processing, computer vision, machine learning, pattern recognition, multimedia databases, and smart sensors. The main mission of this special issue is to identify state-of-the-art machine learning paradigms that are particularly powerful and effective for modeling and combining temporal and spatial media cues such as audio, visual, and face information and for accomplishing tasks of multimedia data mining and knowledge discovery. These models should be able to bridge the gap between low-level audiovisual features which require signal processing and high-level semantics. A number of papers have been submitted to the special issue in the areas of imaging, artificial intelligence; and pattern recognition and five contributions have been selected covering state-of-theart algorithms and advanced related topics.

The first contribution by D. Xiang et al. "Evaluation of data quality and drought monitoring capability of FY$3 A$ MERSI data" describes some basic parameters and major technical indicators of the FY-3A, and evaluates data quality and drought monitoring capability of the MediumResolution Imager (MERSI) onboard the FY-3A.

The second contribution by A. Belatreche et al. "Computing with biologically inspired neural oscillators: application to color image segmentation" investigates the computing capabilities and potential applications of neural oscillators, a biologically inspired neural model, to gray scale and color image segmentation, an important task in image understanding and object recognition. The major contribution of this paper is the ability to use neural oscillators as a learning scheme for solving real world engineering problems.

The third paper by A. Dargazany et al. entitled "Multibandwidth Kernel-based object tracking" explores new methods for object tracking using the mean shift (MS). A bandwidth-handling MS technique is deployed in which the tracker reach the global mode of the density function not requiring a specific staring point. It has been proven via experiments that the Gradual Multibandwidth Mean Shift tracking algorithm can converge faster than the conventional kernel-based object tracking (known as the mean shift).

The fourth contribution by S. Alzu'bi et al. entitled " $3 D$ medical volume segmentation using hybrid multi-resolution statistical approaches" studies new 3D volume segmentation using multiresolution statistical approaches based on discrete wavelet transform and hidden Markov models. This system commonly reduced the percentage error achieved using the traditional 2D segmentation techniques by several percent.

Furthermore, a contribution by G. Cabanes et al. entitled "Unsupervised topographic learning for spatiotemporal data mining" proposes a new unsupervised algorithm, suitable for the analysis of noisy spatiotemporal Radio Frequency Identification (RFID) data. The new unsupervised algorithm depicted in this article is an efficient data mining tool for behavioral studies based on RFID technology. It has the ability to discover and compare stable patterns in a RFID signal, and is appropriate for continuous learning. 
Finally, we would like to thank all those who helped to make this special issue possible, especially the authors and the reviewers of the articles. Our thanks go to the Hindawi staff and personnel, the journal Manager in bringing about the issue and giving us the opportunity to edit this special issue.

Djamel Bouchaffra Abbes Amira

Ce Zhu

Chu-Song Chen 

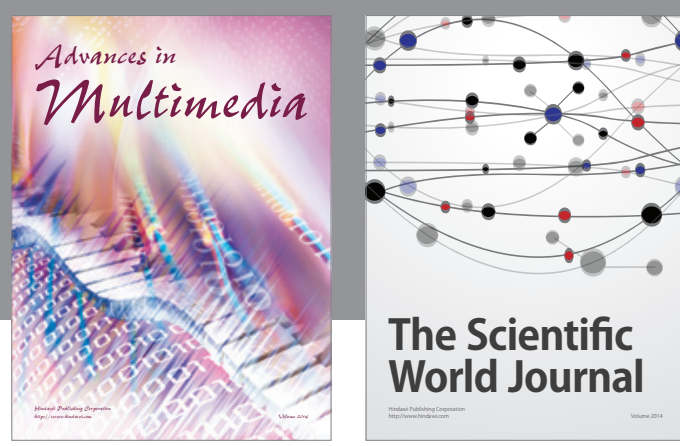

The Scientific World Journal
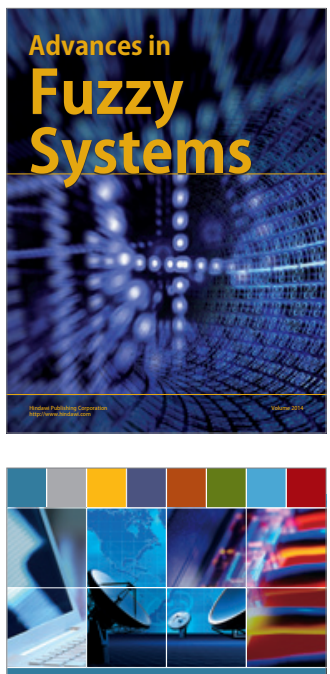

Computer Networks and Communications
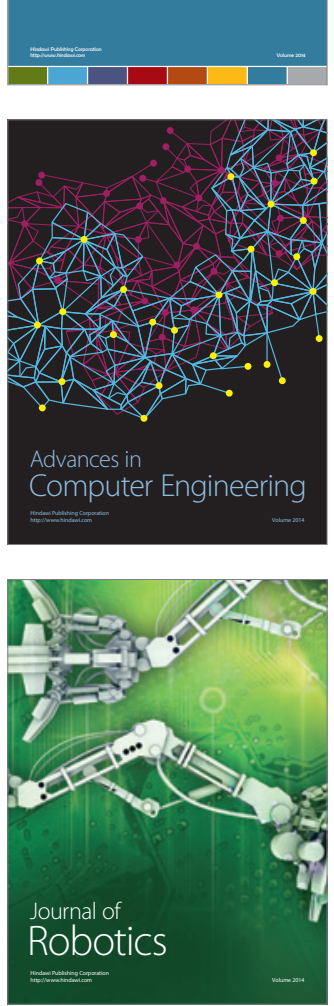
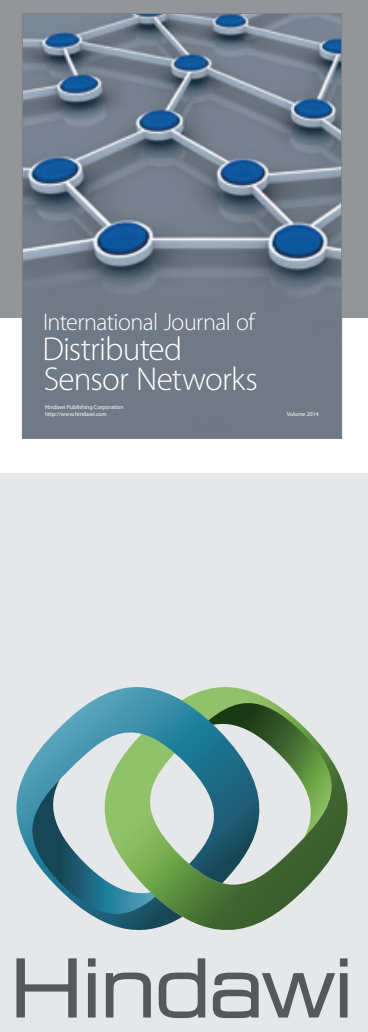

Submit your manuscripts at

http://www.hindawi.com
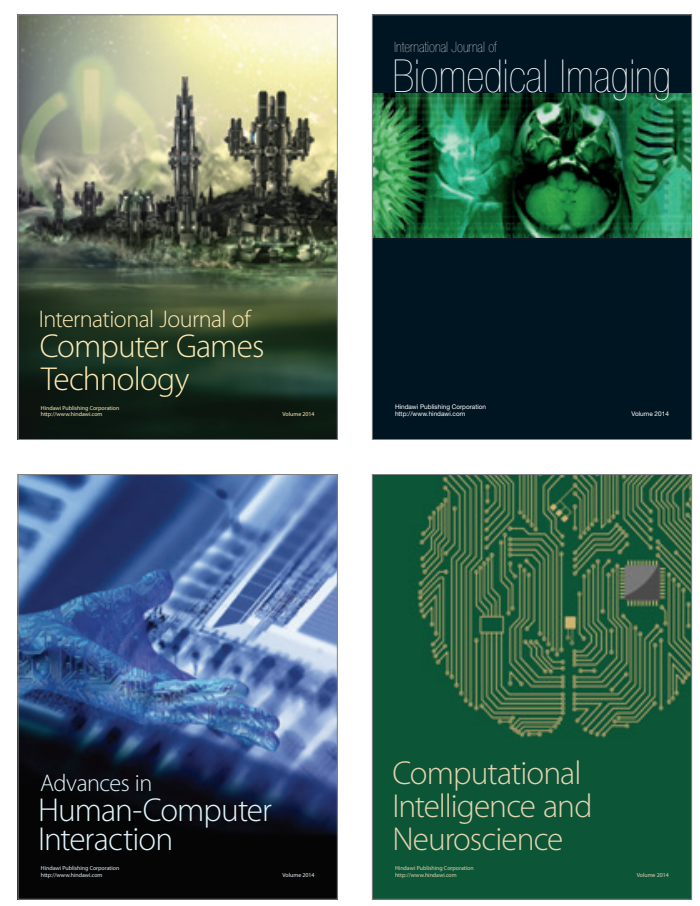
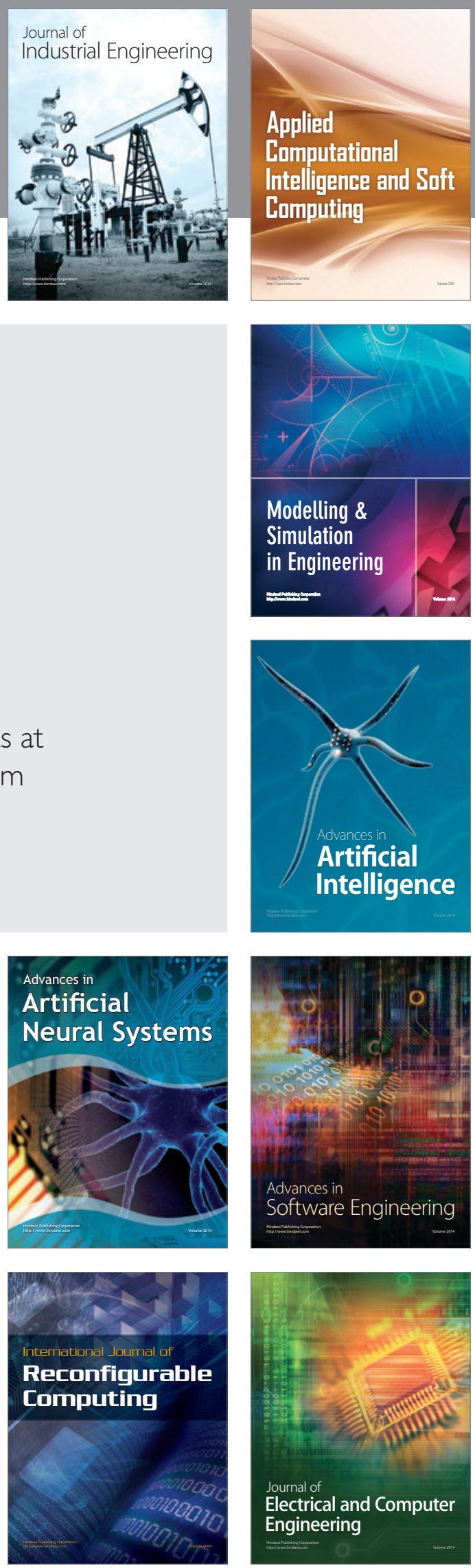